\title{
Point-Contact Study of Fast and Slow Two-Level Fluctuators in Metallic Glasses
}

\author{
R. J.P. Keijsers, ${ }^{1}$ O. I. Shklyarevskii, ${ }^{1,2}$ and H. van Kempen ${ }^{1}$ \\ ${ }^{1}$ Research Institute for Materials, University of Nijmegen, Toernooiveld 1, 6525 ED Nijmegen, The Netherlands \\ ${ }^{2}$ B. Verkin Institute for Low Temperature Physics and Engineering, National Academy of Science of Ukraine, 47 Lenin Avenue, \\ 310164 Kharkov, Ukraine \\ (Received 22 May 1996)
}

\begin{abstract}
Distinct peaks in the differential resistance of point contacts of metallic glasses have been observed around zero bias. These peaks can be attributed to the interaction between conduction electrons and fast switching two-level fluctuators (TLF). Discrete jumps between two such peaks were found to occur, which can be explained by a modulation of the electron-TLF coupling by defects, present in the vicinity of the contact, that slowly switch between two configurations. [S0031-9007(96)01423-8]

PACS numbers: 72.15.Qm, 72.10.Fk, 71.23.Cq, 73.40.Jn
\end{abstract}

The concept of two-level fluctuators (TLF's) was originally proposed to describe many of the low-temperature physical properties of glassy materials (for a review, we refer to Black [1]), but has later on found applications in a much wider field of physics. It was shown, for example, that two-level fluctuators are the dominant source of $1 / f$ noise in metal films [2]. For glassy systems, numerous roughly equivalent, "stable" noncrystalline atomic configurations must exist. Most of these states are too distant to have a crossover from one configuration to another, but some may be accessible within an experimental time scale and are thus constituting a TLF. This general picture suggests that a TLF is a local rearrangement of a small group of atoms. However, even after much research, the exact nature of TLF's is still unknown.

Lately, TLF's have been the subject of several pointcontact (PC) studies. A PC probes only a very small volume (typically $<10^{3} \mathrm{~nm}^{3}$ ), and can therefore be used to study the effect of a few or even a single TLF, present close to the contact area. Beautiful examples of such experiments are observations of two-level resistance fluctuations in clean metallic constrictions caused by lattice defects moving between two metastable configurations $[3,4]$, and of the electromigration of a single defect through a metallic nanoconstruction [5].

Some recent experimental studies of anomalous features of the PC conductance or spectrum $d^{2} V / d I^{2}$ as a function of bias voltage $V_{b}$ in the region of small biases $(<10 \mathrm{mV})$ attributed these anomalies to the presence of TLF's in the constriction region [6-8]. From a PC spectroscopic point of view, a singularity can be expected at $e V_{b}=E_{j}$ when a TLF with a level splitting $E_{j}$ is present close to the PC. Kozub and Kulik (KK) [9] showed that the elastic scattering of electrons from a TLF with different scattering cross sections for both states may lead to a sharp peak in the PC spectrum at small biases. Vladar and Zawadowski (VZ) [10] demonstrated that, at sufficiently low temperatures, a strong coupling between TLF's and conduction electrons may lead to a nonmagnetic Kondolike resonance. This work did not focus on effects due to the PC geometry, whereas $\mathrm{KK}$ did not take into account the collective effects considered by VZ. Both descriptions lead to anomalous features of approximately the same shape, which makes it difficult to distinguish judging from spectral features alone [8]. However, the observation of excellent agreement with conductance scaling predictions for the two-channel Kondo scattering of electrons from TLF's [11] favors the work of VZ. On the other hand, only the KK calculations are able to explain the positive [12] sign of the anomaly that is sometimes observed [8]. For the VZ model, the switching rate of the TLF's should be so large that a conduction electron sees at least a few switching occasions during the electronTLF interaction time. It was suggested [13] that, since the TLF-Kondo interaction is rather sensitive to the exact defect arrangement, a slowly moving TLF may modulate the TLF-Kondo scattering, thus making this slow defect motion observable. A similar effect may occur for the KK description, which mainly concerns relatively slow TLF's [9], e.g., when a rather large defect modulates the number of TLF's from which electrons are elastically scattered when passing through a point contact.

We show in this Letter that a modulation of the electronTLF coupling by slowly moving defects can indeed be observed using point contacts. This requires the presence of many TLF's with a broad distribution of switching times close to the PC. Therefore, materials with a high density of TLF's, such as metallic glasses, are needed.

The experiments were performed using commercially available $1-2 \mathrm{~mm}$ wide and $60-80 \mu \mathrm{m}$ thick ribbons of $\mathrm{Fe}$ - and Ni-based metallic glasses [14] that were cut by laser or abrasive disk, leaving a bridge of less than $0.2 \mathrm{~mm}$ wide. This bridge was narrowed down by filing with a diamond-grain covered wire, after which the ribbon was glued to a phosphor-bronze bending beam covered with a thin layer of insulating kapton foil, leaving the bridge unglued. By bending the beam, one can break the bridge in a clean environment, and can accurately control the contact size between the two freshly exposed electrodes of the thus created, highly stable mechanically controllable 
break (MCB) junction [15]. Some additional experiments were done using silver PC's, for which $50 \mu \mathrm{m}$ thin wires with a deep notch instead of the bridge were used. The PC spectra and conductance curves were recorded using a dc current source and a small $5 \mathrm{kHz}$ current modulation, extracting the second and first harmonic from the voltage signal by means of a conventional lock-in technique.

Figure 1 displays a typical spectrum that is obtained for low Ohmic $(<30 \Omega)$ metallic glass point contacts. The main feature of the PC spectrum at $1.2 \mathrm{~K}$ is a deep negative [12] peak centered around $V_{b}=1 \pm 0.3 \mathrm{mV}$. The shape of this anomalous feature around zero bias does not change notably when magnetic fields up to $5 \mathrm{~T}$ are applied, which rules out the usual magnetic Kondo effect for which Zeeman splitting should be present. The Kondo effect was also ruled out as an explanation for the experimental data on the resistivity of bulk samples of one (METGLAS 2826A) of the metallic glasses studied here [16].

The peak in the PC spectrum corresponds to a rather narrow maximum in the differential resistance $R_{d}$ around zero bias (see, e.g., curve 1 in Fig. 2). The decrease of $R_{d}$ away from zero bias, associated with the PC spectrum peak, is usually in the range of $(1-3) \%$ but can reach values of $(5-10) \%$ for the Ni-based metallic glasses. This is over an order of magnitude larger than what has been found for normal metal PC's $[8,13]$. In the range of $1.5-5 \mathrm{mV}$, the peak shape is described quite nicely by both $\mathrm{VZ}$ and $\mathrm{KK}$, indicating that the spectrum is here mainly determined by electron-TLF scattering. However, above 5-6 $\mathrm{mV}, R_{d}$ decreases proportional to $V_{b}^{0.5}$ or $V_{b}$, depending on the specific metallic glass. This is not in agreement with the behavior expected for both $\mathrm{VZ}$ and

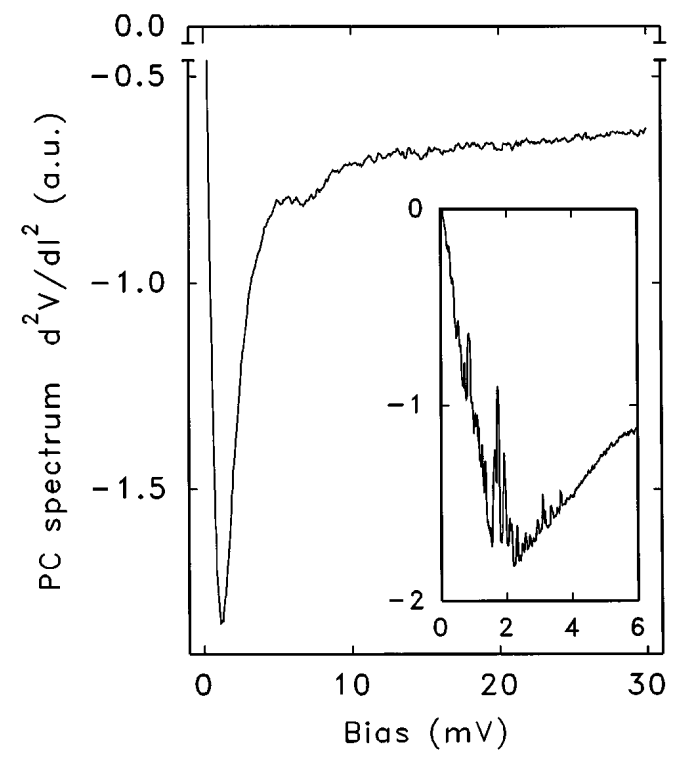

FIG. 1. Example of the point-contact spectrum of a low Ohmic $\mathrm{Fe}_{80} \mathrm{~B}_{20}$ metallic glass MCB junction $(T=1.2 \mathrm{~K}, R=$ $16 \Omega$ ). The inset shows the peak minimum for a rather high Ohmic $(65 \Omega)$ contact.
$\mathrm{KK}$, indicating that other scattering mechanisms play a dominant role here [17].

Two effects were observed as the contact resistance was increased above 30-50 $\Omega$ (see the inset of Fig. 1). First of all, the peak close to zero bias was found to smear out, and the position of the minimum shifted towards higher biases. This effect may be attributed to the fact that the size of the defects to which the TLF's are related can become comparable to the contact size for high Ohmic contacts. Because the ratios of the inelastic and elastic electron mean free paths to the contact diameter increase for smaller contacts, it is also possible that other scattering mechanisms (such as electron-electron scattering) become important at small biases.

Furthermore, the high Ohmic contacts displayed large fluctuations of the second harmonic signal, which sometimes made it impossible to record the spectra for small bias voltages. Slow registration of the differential resistance (with a time constant of $0.03 \mathrm{~s}$ ) showed that this effect is connected to low-frequency, steplike fluctuations of $R_{d}$. Low Ohmic contacts reveal only very weak lowfrequency fluctuations at small biases (curve 1 in Fig. 2), whereas high Ohmic contacts demonstrate jumps in $R_{d}$ that have a maximal amplitude (up to $0.5 \%$ of $R_{d}$ ) around zero bias, decreasing to the level of the background noise mostly at $V=2-3 \mathrm{mV}$, but sometimes not before $V>$ $5 \mathrm{mV}$ [curves 2 and 3 in Figs. 2 and 3(a)]. This behavior is in sharp contrast to the usual increase of the PC noise level with the applied bias voltage.

Curve 3 in Fig. 2 displays reversible jumps between two slightly different differential resistance peaks around zero bias. As stated above, it was suggested by Ralph and

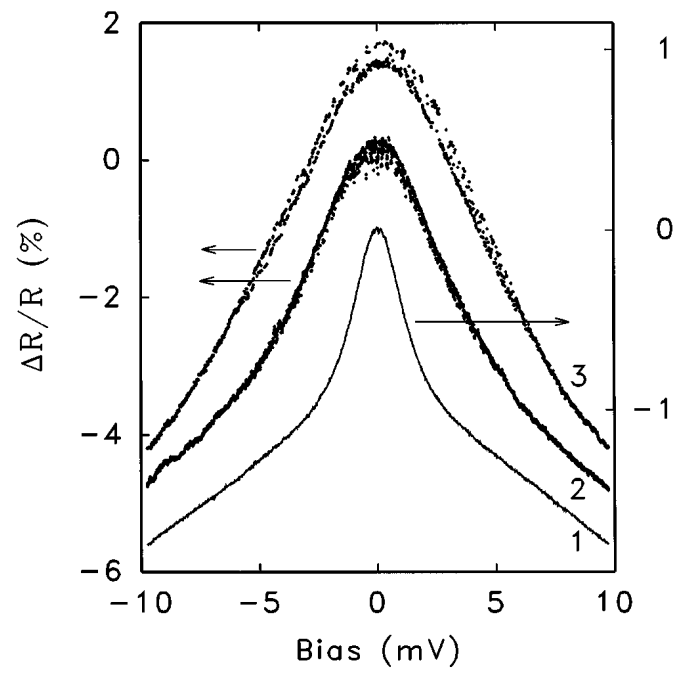

FIG. 2. Differential resistance $R_{d}$ as a function of bias voltage for $\mathrm{Fe}_{80} \mathrm{~B}_{20}$ (1 and 2) and $\mathrm{Fe}_{32} \mathrm{Ni}_{36} \mathrm{Cr}_{14} \mathrm{P}_{12} \mathrm{~B}_{6}$ (3) MCB junctions $(T=1.2 \mathrm{~K})$. (1) A $6.6 \Omega$ contact, displaying almost no noise. (2) A $366 \Omega$ contact that shows clear noise around zero bias. The noise amplitude decreases as the bias voltage increases. (3) A $145 \Omega$ contact, showing a clear two-level switching behavior between two different $R_{d}$ peaks. 

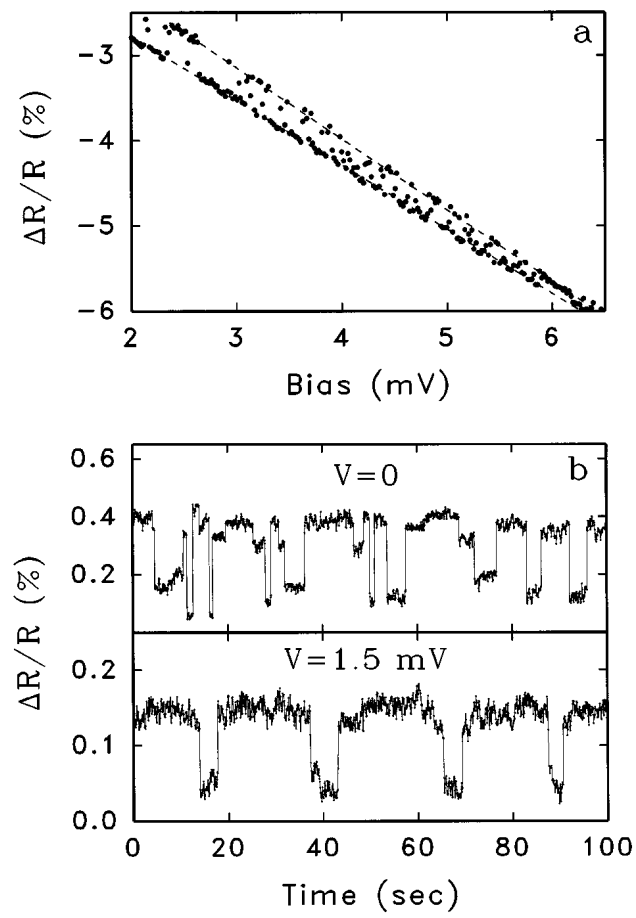

FIG. 3. (a) Enlargement of a part of the third curve of Fig. 2, clearly showing two-level jumps in $R_{d}$ with an amplitude that decreases as the bias voltage increases. (b) Differential resistance $R_{d}$, recorded as a function of time for a $366 \Omega$ $\mathrm{Fe}_{80} \mathrm{~B}_{20} \mathrm{MCB}$ junction at bias voltages of 0 and $1.5 \mathrm{mV}$. At zero bias, jumps between several levels occur, but, at $1.5 \mathrm{mV}$, only two resistance levels are present $(T=1.2 \mathrm{~K})$.

Buhrman [13] that a slowly moving defect (e.g., a cluster of atoms, or a dislocation) in the vicinity of the contact area may cause the nonmagnetic Kondo-like electron-TLF coupling to switch between two configurations. Experimentally, this would lead to reversible jumps between two slightly different differential resistance peaks around zero bias, which immediately explains the decrease of the amplitude of the low-frequency telegraph-noise-like behavior of $R_{d}$ with increasing bias voltage [Fig. 3(a)]. When the slow motion of a large defect changes the number of TLF's that are probed by the point contact, or certain parameters (e.g., the electron scattering cross section of one or both states of the TLF) of a part of them, switches between two slightly different differential resistance peaks may also occur within the framework of the KK calculations. We therefore believe that the observed instability of the contact resistance is a direct result of a modulation of the electron-TLF coupling by slowly moving defects.

Here we note that any mechanism that causes such a distinct structure in the spectrum in a narrow range around zero bias and that is sensitive to the switching of a slowly moving defect in the vicinity of the constriction may lead to a similar contact resistance instability. For the differential resistance peak in point-contact experiments two causes are discussed in the literature [18]: Kondo effect and electron-TLS interaction. However, in spectroscopic electron tunneling studies, zero-bias peaks have also been related to Coulomb blockade effects and to density of states effects. Coulomb blockade effects require a localized charge and are not expected to appear as a common feature in a point-contact geometry where there is a direct connection between the two electrodes. (It is not even common in scanning tunneling microscopy.) A dip in the density of states near the Fermi level is known to occur due to electron-electron interactions. However, the related conductance reduction is theoretically expected to be, at most, of the order of $e^{2} / h$ [13] and cannot account for the conductance reduction of about 100 times this value for the $6.6 \Omega$ sample in Fig. 2. As was stated above, the differential resistance peak cannot result from the usual Kondo effect, which leaves electron-TLS interaction as the only known cause for the point-contact $R_{d}$ peak.

Generally, more than one modulating defect was present. In some cases, the number of active defects (or, alternatively, the number of accessible levels for one large defect) changed with applied bias. An example of this behavior is given in Fig. 3(b). Here, time traces of $R_{d}$ for a $366 \Omega \mathrm{Fe}_{80} \mathrm{~B}_{20}$ contact display jumps between a few distinct levels at zero-bias voltage, whereas at $1.5 \mathrm{mV}$ only one defect switching between two states remains active.

For all contacts, the noise level at higher frequencies, registered with a storage oscilloscope using a bandpass of $0-30 \mathrm{kHz}$, stayed nearly the same or increased slightly as the bias voltage was increased from 0 to $10 \mathrm{mV}$, indicating that faster fluctuations in this frequency range do not, or only to a negligibly small level, affect the electron-TLF coupling. Because of the amorphous nature of the material under study, one would expect that TLF's over a broad range of frequencies should be present. It is very likely that a switching event from one state to the other of a TLF will be easier when the number of atoms involved is smaller, i.e., faster TLF's have a smaller size. This explains why only modulation by very lowfrequency TLF's has been observed, because they are, due to their size, much more likely to affect notably the electron-TLF coupling.

In a normal metal, the density of TLF's is approximately 2 orders of magnitude smaller [13] than for metallic glasses, and often there will be no slowly switching defects present in the immediate vicinity of the contact, which makes an observation of the effects described above unlikely. It is therefore not unexpected that the PC spectra of Ag MCB junctions, recorded after a low-temperature break, show little or no zero-bias fluctuations for most of the studied contacts. However, occasionally, $R_{d}$ is very noisy for small bias voltages, with the noise level decreasing with increasing bias voltage (curve 1 of Fig. 4). In these cases, the relative amplitude of the noise is much higher than for metallic glasses, which can already originate from the fact that $\mathrm{Ag}$ contacts with the same contact resistance as a metallic glass contact have a much 
smaller size (3-4 nm for a $100 \Omega$ contact), and the fluctuation amplitude tends to increase with decreasing contact size. The noise also extends to larger bias voltages, and the fluctuations are much faster. Apparently, the defect motion can be observed to much higher frequencies here than for metallic glasses, which may again be connected to the smaller size of the Ag contacts that leads to a stronger effect of defect rearrangement on the electron scattering on fast TLF's. The distortion of the zero-bias peak at higher voltages in Fig. 4 is due to the occurrence of electron-phonon interaction. Annealing of the Ag junction at room temperature results, for high Ohmic contacts, in a reduction of the zero-bias peak intensity (curve 2 of Fig. 4), indicating a decrease of the number of fast TLF's. However, slow zero-bias resistance fluctuations were never observed for these "annealed" samples, which implies a complete disappearance of slowly switching defects.

In conclusion, using point contacts of metallic glasses, we have observed distinct peaks around zero bias in the differential resistance, which can be attributed to the interaction between conduction electrons and TLF's. Discrete jumps between two (or more) of such peaks in the differential resistance occurred. This can be explained by considering defects in the vicinity of the contact, slowly switching between two configurations, which cause a modulation of the interaction between conduction electrons and the TLF's in the vicinity of the contact. The experimental results do not provide unambiguous evidence in favor of either the $\mathrm{KK}$ or the VZ model describing the resistance peak. Both mechanisms may even work simultaneously in amorphous

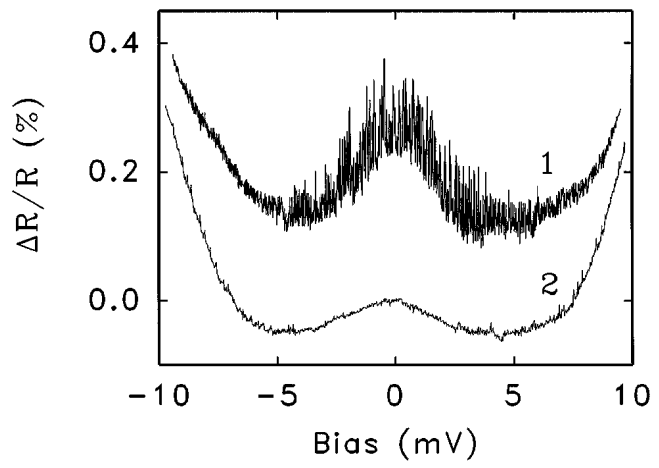

FIG. 4. Differential resistance $R_{d}$ as a function of bias voltage for an $\mathrm{Ag} \mathrm{MCB}$ junction $(T=1.2 \mathrm{~K})$. (a) A $100 \Omega \mathrm{MCB}$ junction after a low-temperature break. The noise level is very high over a rather wide bias voltage range. (2) Again, a $100 \Omega$ junction, after annealing the sample in vacuum at room temperature. The zero-bias peak is reduced, and the noise has completely disappeared. solids. At higher bias voltages, clear deviations from both descriptions are found, indicating that other electron scattering mechanisms are important here. The measurements thus give an indication of the energy range where strong electron-TLF coupling occurs.

Part of this work was supported by the Stichting voor Fundamenteel Onderzoek der Materie (FOM) which is financially supported by the Nederlandse Organisatie voor Weterschappelijk Onderzoek (NWO). We thank J. G. H. Hermsen for technical support, and I. K. Yanson and Yu. A. Kolesnichenko for stimulating discussions. O.I.S. wishes to acknowledge the NWO for a visitor's grant.

[1] J. L. Black, in Glassy Metals I, edited by H. J. Güntherodt and H. Beck (Springer-Verlag, Berlin, 1981), p. 167.

[2] K. S. Ralls and R.A. Buhrman, Phys. Rev. B 44, 5800 (1991).

[3] K. S. Ralls and R. A. Buhrman, Phys. Rev. Lett. 60, 2434 (1988).

[4] P.A.M. Holweg, J. Caro, A.H. Verbruggen, and S. Radelaar, Phys. Rev. B 45, 9311 (1992).

[5] K. S. Ralls, D. C. Ralph, and R. A. Buhrman, Phys. Rev. B 40, 11561 (1989).

[6] D. C. Ralph and R. A. Buhrman, Phys. Rev. Lett. 69, 2118 (1992).

[7] A. I. Akimenko and V.A. Gudimenko, Solid State Commun. 87, 925 (1993).

[8] R. J.P. Keijsers, O. I. Shklyarevskii, and H. van Kempen, Phys. Rev. B 51, 5628 (1995).

[9] V.I. Kozub and I. O. Kulik, Zh. Eksp. Teor. Fiz. 91, 2243 (1986) [Sov. Phys. JETP 64, 1332 (1986)].

[10] K. Vladar and A. Zawadowski, Phys. Rev. B 28, 1564 (1983); 28, 1582 (1983); 28, 1596 (1983).

[11] D. C. Ralph, A. W.W. Ludwig, J. von Delft, and R. A. Buhrman, Phys. Rev. Lett. 72, 1064 (1994).

[12] We define the sign of the low-bias peak relative to the sign of the electron-phonon interaction spectra.

[13] D. C. Ralph and R. A. Buhrman, Phys. Rev. B 51, 3554 (1995).

[14] We used $\mathrm{Fe}_{80} \mathrm{~B}_{20}, \quad \mathrm{Fe}_{78} \mathrm{Mo}_{2} \mathrm{~B}_{20}, \quad \mathrm{Fe}_{40} \mathrm{Ni}_{40} \mathrm{P}_{14} \mathrm{~B}_{6}$, and $\mathrm{Fe}_{32} \mathrm{Ni}_{36} \mathrm{Cr}_{14} \mathrm{P}_{12} \mathrm{~B}_{6}$ (METGLAS 2605, 2605A, 2826, and 2826A, produced by Allied-Signal, Inc.).

[15] C. J. Muller, J. M. van Ruitenbeek, and L. J. de Jongh, Physica (Amsterdam) 191C, 485 (1992).

[16] R. W. Cochrane, R. Harris, J. O. Ström-Olson, and M. J. Zuckermann, Phys. Rev. Lett. 35, 676 (1975).

[17] R. J. P. Keijsers, O. I. Shklyarevskii, and H. van Kempen (to be published).

[18] I. K. Yanson and O. I. Shklyarevskii, Fiz. Nizk. Temp. 12, 899 (1986) [Sov. J. Low Temp. Phys. 12, 509 (1986). 